\title{
NON-NEWTONIAN MOMENTUM TRANSFER PAST AN ISOTHERMAL STRETCHING SHEET WITH APPLIED SUCTION
}

\author{
P.H. VEENA* \\ Dept. of Mathematics, Smt. V.G. College for Women \\ Gulbarga Karnataka, INDIA \\ E-mail: drveenaph@yahoo.com \\ B. SURESH \\ Research Scholar, Dept. of Mathematics Gulbarga, University Gulbarga \\ Karnataka, INDIA \\ V.K. PRAVIN \\ Dept. of Mech. Engg., P.D.A. College of Engg. \\ Gulbarga, Karnataka, INDIA \\ A.M. GOUD \\ Dept. of Mathematics, A S M College for Women \\ Bellary, Karnataka, INDIA

\begin{abstract}
The paper discusses the flow of an incompressible non-Newtonian fluid due to stretching of a plane elastic surface in a saturated porous medium in the approximation of boundary layer theory. An exact analytical solution of non-linear MHD momentum equation governing the self-similar flow is given. The skin friction co-efficient decreases with an increase in the visco-elastic parameter $k_{l}$ and increase in the values of both the magnetic parameter and permeability parameter.
\end{abstract}

Key words: non-Newtonian fluid, magnetic field, permeability, effect of viscoelasticity.

\section{Introduction}

The study of a magneto convection flow of non-Newtonian fluids over a continuously moving porous wall has wide applications in technological and manufacturing processes in industries. To be more specific, it may be pointed out that many metallurgical processes involve the cooling of continuous strips or filaments by drawing them through a quiescent fluid and that in the process of drawing these strips are sometimes stretched.

The numerous applications of non-Newtonian fluids have led to a renewed interest among researchers to investigate the visco-elastic boundary layer flow over a stretching plastic sheet Rajgopal et al. [1], Dandapat et al. [2], Rollins and Vajravelu [3], Anderson [4], Lawrence and Rao [5], Char [6] considered the motion of power law fluid flow past a stretching sheet. The Rivlin Erickson fluid was studied by Siddappa and Khapate [7], the Walters liquid B' was considered by Siddappa and Abel [8], Veena et al. [911] which the fluids was studied exhibit normal stress differences in simple shear flows. A great deal of literature is available including those cited above on the two-dimensional visco-elastic boundary layer flow over a stretching surface where the velocity of the stretching surface is assumed linearly proportional to the

\footnotetext{
* To whom correspondence should be addressed
} 
distance from a fixed origin. However, Gupta and Gupta [12] pointed out that realistically stretching of the sheet may not necessarily by linear.

This situation was investigated by Kumaran and Ramanaiah [13] in their work on a boundary layer flow over a quadratic stretching sheet. But their work was confined to the viscous flow past a stretching sheet. McCormack and Crane [14] provided a comprehensive discussion on a boundary layer flow caused by stretching of an elastic flat sheet moving in its own plane with a velocity varying linearly with distance. Rajgopal et al. [15] analyzed the effects of visco-elasticity on the flow of a second order fluid with gradually fading memory and they arrived at the same governing boundary layer equation as that in $(2,3,9,11,12$ and 13). The influence of a uniform transverse magnetic field on the motion of an electrically conducting fluid past a stretching surface was studied by Pavlov [16]. An MHD flow of visoc-elastic fluids was probably first considered by Sarpakaya [17], while Anderson [4] and Dandapat et al. [18] obtained the similarity solutions of the boundary layer equation governing the flow in an elastic power-law fluid in the presence of an external magnetic field.

Abel et al. [19], Veena et al. [20], Veena et al. [21] obtained the non-similar solutions of viscous and visco-elastic boundary layer flows with suction blowing, steady and unsteady aspects with porosity and a magnetic field past continuously moving stretching bodies. Khan and Sanjayanand [22] studied the viscoelastic boundary layer flows and heat transfer over an exponential stretching sheet. Sajid et al. [23], investigated the non-similar analytical solutions for an MHD flow and heat transfer in a third order fluid past a stretching sheet. Abel et al. [24], analyzed the MHD flow of a visco-elastic fluid over a stretching sheet. Pantokratoras [25] made a numerical investigation of an MHD boundary layer flow with variable viscosity past a stretching surface. Motivated by all the above analyses in the present note we are concerned with the study of combined effects of visco-elasticity, the magnetic field and porous parameter over a continuously moving stretching surface in the presence of suction.

\section{Flow analysis}

An incompressible second-order fluid has a constitutive equation based on the assumptions of the principle given by Coleman and Noll [26] as

$$
T=-p I+\mu A_{1}+\alpha_{1} A_{2}+\alpha_{2} A_{1}^{2}
$$

where $T$ is the stress tensor, $p$ is the pressure, $\mu, \alpha_{1}, \alpha_{2}$ are the material constants with $\alpha_{1}<0$ and $A_{1,} A_{2}$ are defined as

$$
\begin{aligned}
& A_{1}=\operatorname{grad} v+(\operatorname{grad} v)^{T}, \\
& A_{2}=\frac{d}{d t} A_{1}+A_{1} \cdot \operatorname{grad} v+(\operatorname{grad} v)^{T} \cdot A_{1} .
\end{aligned}
$$

We considered the flow of a fluid obeying the constitution Eq.(2.1) which displays normal stress differences in the shear flow and is an approximation to a simple fluid in the sense of retardation. This model is applicable to some dilute polymer solutions and is valid at low rates of shear.

The fluid flow obeying (2.1) past a flat porous sheet coinciding with the plane $y=0$, the flow is confined to $y>0$. Two equal and opposite forces are applied along the $x$-axis. So the wall is stretched keeping the origin fixed in a uniform magnetic field. 


\section{Physical sketch}

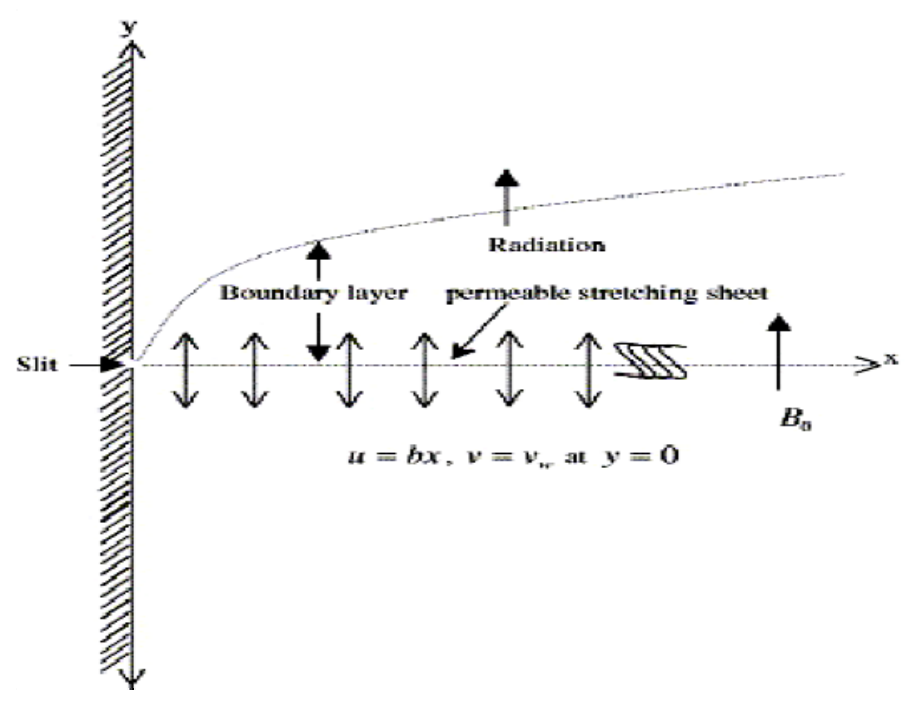

The steady incompressible two dimensional boundary layer equations of motion for visco-elastic Walters liquid B' in the presence of a magnetic field and a porous medium obtained by Bread and Walters [27] in usual notation are

$$
\begin{aligned}
& \frac{\partial u}{\partial x}+\frac{\partial v}{\partial y}=0, \\
& u \frac{\partial u}{\partial x}+v \frac{\partial u}{\partial y}-v \frac{\partial^{2} u}{\partial y^{2}}+k\left[\frac{\partial}{\partial x}\left(u \frac{\partial^{2} u}{\partial y^{2}}\right)+\frac{\partial u}{\partial y} \frac{\partial^{2} v}{\partial y^{2}}+v \frac{\partial^{3} v}{\partial y^{3}}\right]-\frac{v}{k^{\prime}} u-\frac{\sigma B_{0}^{2}}{\rho} u=0,
\end{aligned}
$$

where $\quad v=\frac{\mu}{\rho}, \quad k=-\frac{\alpha_{1}}{\rho}$.

In deriving the above equations it is assumed that the normal stress is of the same order of magnitude as that due to shear stresses. Thus both $v, k$ are of $O\left(\delta^{2}\right), \delta$ being the boundary layer thickness.

The appropriate boundary conditions are

$$
\begin{aligned}
& u=b x, \quad v=V_{w} \quad \text { at } \quad y=0, \\
& u \rightarrow 0 \quad \text { as } \quad y \rightarrow \infty, \quad b>0 \quad \text { ( } b \text { is the stretching rate) }
\end{aligned}
$$

The flow is caused solely by the stretching of the wall and the free stream velocity being zero. Equations (3.1) and (3.2) admit self-similar solution of the type.

$$
u=b x f_{\eta}(\eta): \quad v=-(b v)^{\frac{1}{2}} f(\eta) ; \quad \eta=\left(\frac{b}{v}\right)^{\frac{1}{2}} y .
$$

The equation of continuity is satisfied identically and substituting Eq.(3.5) in Eq.(3.2) it converts to 


$$
\begin{aligned}
& f_{\eta}{ }^{2}(\eta)-f(\eta) f_{\eta \eta}(\eta)-f_{\eta \eta \eta}(\eta)+k_{l}\left[2 f_{\eta}(\eta) f_{\eta \eta \eta}(\eta)-f_{\eta \eta}{ }^{2}(\eta)-f(\eta) f_{\eta \eta \eta}(\eta)\right]+ \\
& +\left(k_{2}+M_{n}\right) f_{\eta}(\eta)=0
\end{aligned}
$$

where the suffix $\eta$ denotes differentiation with respect to $\eta$ and

$$
k_{1}=\frac{k b}{v} ; \quad k_{2}=\frac{v}{k^{\prime} b} ; \quad M_{n}=\frac{\sigma B_{0}^{2}}{\rho b},
$$

and velocity components given in Eq.(3.5)

$$
f_{\eta}(0)=1, \quad f(0)=\frac{-V_{w}}{\sqrt{b v}} ; \quad f_{\eta}(\infty)=0, \quad f_{\eta \eta}(0)=0
$$

Equation (3.6) subjected to the three boundary conditions (3.8) was derived by Char [6]. But since Eq.(3.6) is of fourth order and highly non-linear, is satisfies only three boundary conditions of Eq.(3.8). This difficulty was overcome in [6] by expanding $f(\eta)$ in a power series in terms of the visco-elastic parameter $k_{l}$, assuming $k_{l}$ as very small. This of course is valid for dilute polymer solutions.

Hence, we observed one more boundary condition included in Eq.(3.8) to get the unique exact analytical solution of Eq.(3.6) that is $f_{\eta \eta}(0)=0$.

\section{Analytical solution}

Thus it is interesting to note that Eq.(3.6) has a solution of the form

$$
f_{\eta}(\eta)=\exp (-\alpha \eta), \quad \alpha>0
$$

satisfying the boundary conditions $f_{\eta}(0)=1, f_{\eta}(\infty)=0$ and $f_{\eta \eta}(0)=0$ in Eg.(3.8).

Integrating Eq.(4.1) and using

$$
f(0)=R=\frac{-V_{w}}{\sqrt{b v}}, \text { the suction parameter, }
$$

we obtain the non-dimensional velocity variable $f(\eta)$ as

$$
f(\eta)=\frac{1-\exp (-\alpha \eta)}{\alpha}-\frac{-V_{w}}{\sqrt{b v}}
$$

where $\alpha$ is the positive root of the cubic equation

$$
\alpha^{3}-A_{3} \alpha^{2}+A_{3} \alpha-A_{5}=0
$$

and it is found by Graffe's root square method.

Thus from Eq.(4.2) we get a remarkably simple exact analytical solution of Eq.(3.6) satisfying the boundary conditions (3.8). This gives the new velocity components $u$ and $v$ as 


$$
u=b x \exp (-\alpha \eta) ; \quad v=-\sqrt{\frac{b \mu}{\rho}}\left[\frac{1-\exp (-\alpha \eta)}{\alpha}-R\right]
$$

where ' $\alpha$ ' is calculated from the Eq.(4.3) as

$$
\alpha=\left[\frac{1+M_{n}+k_{2}}{1-k_{1}}\right]^{1 / 2} .
$$

The dimensionless shear stress $\tau$ at the wall is obtained as

$$
\begin{aligned}
& \tau=-\mu\left(\frac{\partial u}{\partial y}\right)_{y=0}=f_{\eta \eta}(0)=\left[\frac{1-k_{1}}{1+M_{n}+k_{2}}\right]^{1 / 2}, \\
& \tau=-\mu\left[\alpha^{2} b x\right]
\end{aligned}
$$

which shows that $\tau$ vanishes when $k_{1}=1$. But this can never occur since the solution includes the combined effects of visco-elastic and magnetic forces.

\section{Results and discussion}

To explain the effect of various physical concepts such as boundary layer thickness $\delta$, stream wise velocity, wall friction co-efficient $\tau$, visco-elasticity, permeability and magnetic parameter, many graphs are drawn and discussed as follows.

A momentum boundary layer flow of a visco-elastic fluid past an exponentially stretching impermeable sheet has been investigated in the present study. The governing basic boundary layer equation of momentum is highly non-linear and converted into an ordinary differential equation by applying suitable similarity transformation.

Figure 1 is the graph of velocity profiles $f(\eta)$ and $f_{\eta}(\eta)$ for various values of the visco-elastic parameter for fixed values $M n$ and $k_{2}, R$. It is observed from the figure that the transverse flow velocity $f(\eta)$ is a decreasing function and longitudinal flow velocity $f_{\eta}(\eta)$ is an increasing function of the visco-elastic parameter $k_{1}$.

Figures 2 and 3 depict the graphs of $f(\eta)$ and $f_{\eta}(\eta)$ versus $\eta$ for different values of permeability parameter $k_{2}$ and magnetic parameter $M n$, respectively. In both the cases, the velocity distribution $f(\eta)$ along the transverse direction is a decreasing function of $k_{2}$ and $M n$ where the velocity profile $f_{\eta}(\eta)$ along longitudinal direction is an increasing function of $k_{2}$ and $M n$ respectively for the value of the suction parameter $R=-0.424$.

Figure 4 demonstrates the graph of the skin friction co-efficient versus the visco-elastic parameter $k_{l}$ for fixed values of $k_{2}, R$ and $M n$. From the figure it is noticed that the increase in $k_{1}$ leads to the decrease of skin friction. This is due to the fact that the elastic property in the visco-elastic fluid reduces the frictional force. This result has great significance in polymer processing industry, as the choice of a higher order viscoelastic (Walters liquid B') fluid would reduce the power consumption for stretching the boundary sheet. 


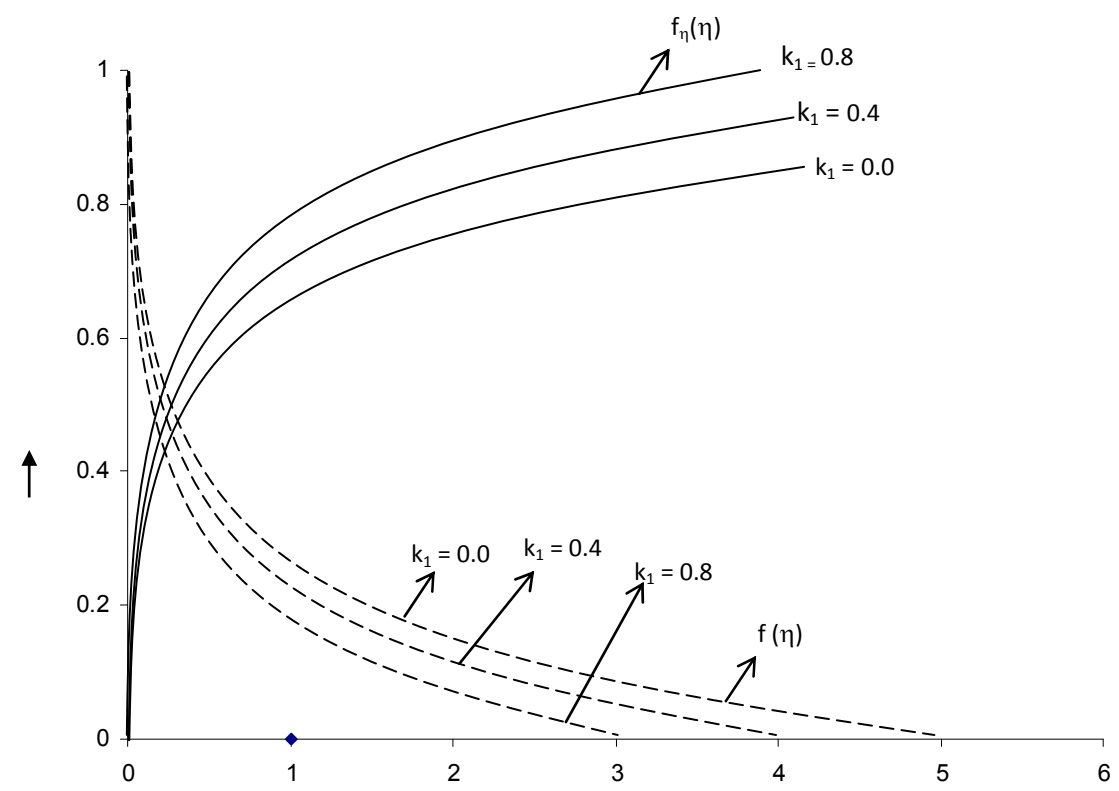

Fig.1. Velocity variation in longitudinal and transverse directions for various values of the visco-elastic parameter $k_{l}$ and fixed values of $k_{2}=2$ and $M n=2$, suction parameter $R=-0.424$.

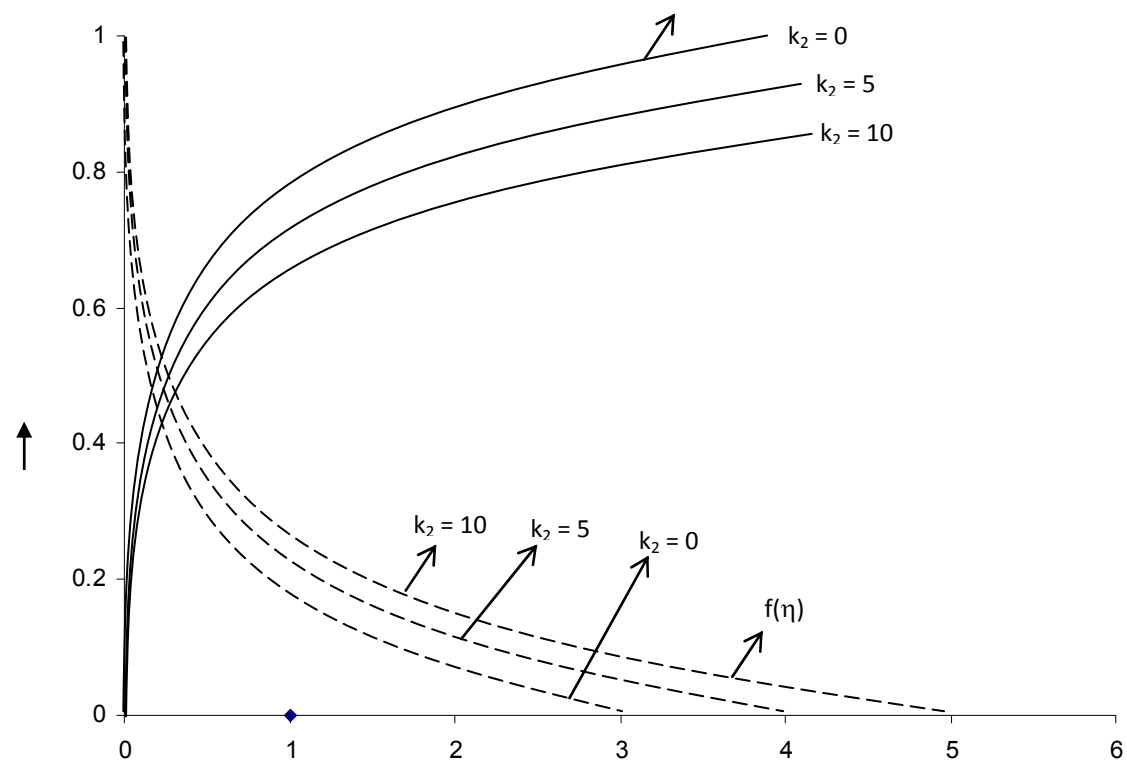

Fig.2. Velocity profiles $f(\eta)$ and $f_{\eta}(\eta)$ vs. $\eta$ for various values of the permeability parameter $k_{2}=0,5,10$ and fixed values of $k_{1}=0.4, M n=2, R=-0.424$. 


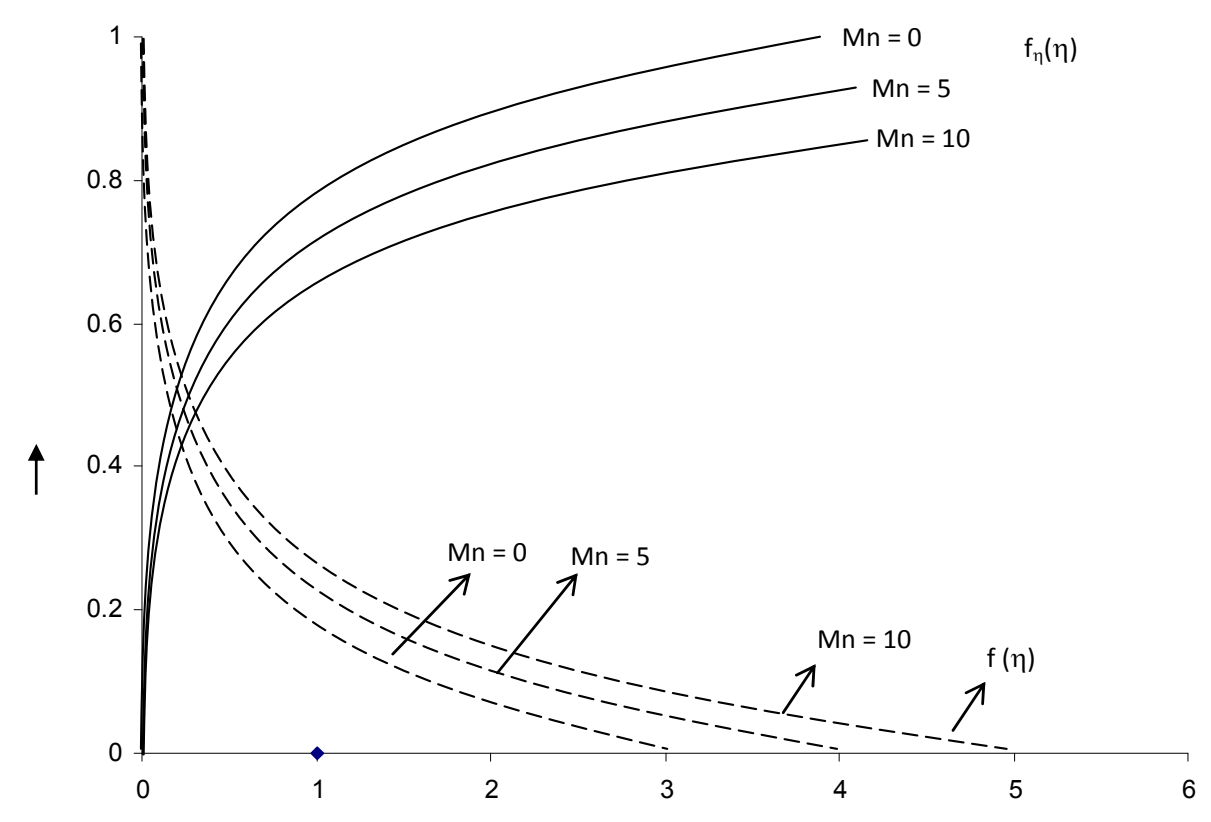

Fig.3. Velocity distribution of $f(\eta)$ and $f_{\eta}(\eta)$ Vs. $\eta$ for different values of $M n=0,5,10$ and fixed values of $k_{1}=0.4, k_{2}=5, R=-0.424$.

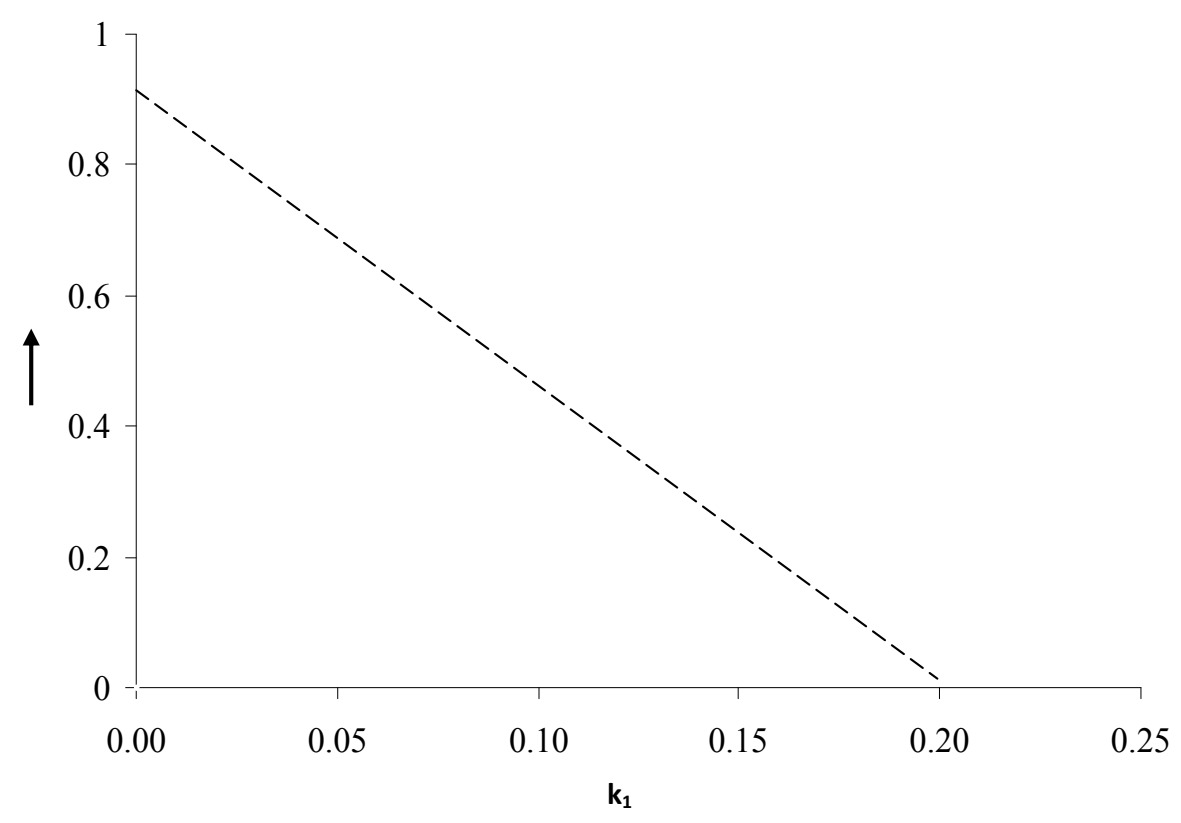

Fig.4. Variation of the skin friction co-efficient $\tau$ vs. the visco elastic parameter $k_{1}$ for values of $k_{2}=5, M n$ $=5$, and $R=0.424$.

\section{Conclusions}

The characteristic feature of the above exact analytical results is that the effect of viscoelasticity, magnetic field and porosity are combined into a single parameter $\alpha \geqq 1$ with added suction $V_{w}$ which is defined in Eq.(4.5). Since themagnetic parameter $M n$ and the permeability parameter $k_{2}$ are 
positive $M n \geq k_{2} \geq 0$ and $0 \leq k_{1} \leq 1$, it can be concluded that an increase in values of $M n$ and $k_{2}$ has the same influence on the flow field as increased velocity. According to Eqs (4.4) and (4.6) the main effects of visco-elasticity, magnetic field and permeability are to reduce the velocity within the boundary layer and the external velocity normal to the sheet and also to reduce the boundary layer thickness while increasing the skin friction.

\section{Nomenclature}

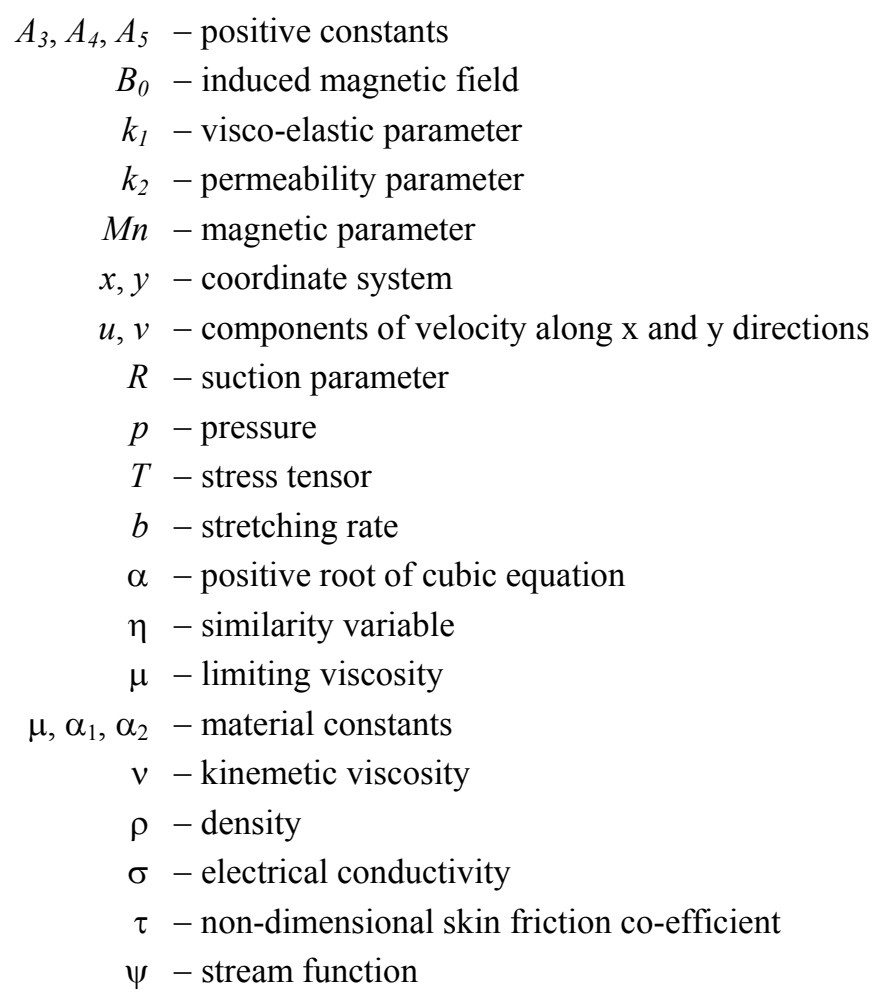

\section{References}

[1] Rajgopal K.R., Na T.Y. and Gupta A.S. (1984): Flow of a visco-elastic fluid over a stretching sheet. - Rheol. Acta., vol.23, pp.213-215.

[2] Dandapat B.S. and Gupta A.S. (1989) : Flow and heat transfer in a visco-elastic fluid over a stretching sheet. - Int. J. Non-Linear Mech., vol.24, No.3, pp.215-219.

[3] Rollins D. and Vajravelu K. (1991): Heat transfer in second order fluid over a continuous stretching surface. Acta. Mech., vol.89, pp.167-178.

[4] Anderson H.I. (1992): MHD flow of a visco-elastic fluid past a stretching surface. - Acta. Mech., vol.95, pp.227-230.

[5] Lawrence P.S. and Rao B.N. (1992): Heat transfer in the flow of visco-elastic fluid over a stretching sheet. - Acta. Mech., vol.93, pp.53-61.

[6] Char M.I. (1994): Heat and mass transfer in a hydro magnetic flow of visco-elastic fluid over a stretching sheet. J. Math. Anal. Appl., vol.186, pp.674-689. 
[7] Siddappa B. and Khapate B.K. (1976): Rivlin-Erickson fluid flow past a stretching plate. - Rev. Roum. Sci. Techn. Mec. Appl., vol.21, pp.497-505.

[8] Siddappa B. and Abel M.S. (1986): Visco-elastic boundary layer flow past a stretching plate with suction and heat transfer. - Rheol. Acta., vol.25, pp.319-320.

[9] Veena P.H., Pravin V.K. and Ashok K. (2009): MHD flow of a vsico-elastic fluid over a stretching sheet with power low surface temperature and power law concentration embedded in a porous medium. - Pacific-Asian J. of Mathematics, vol.3, No.1-2, pp.1-15.

[10] Veena P.H., Pravin V.K. and Shahjahan S.M. (2008): Heat and mass transfer flow with variable thermal conductivity and variable mass diffusivity in an MHD visco-elastic fluid over a non-isothermal stretching sheet. IJAME, vol.13, No.2, pp.515-541.

[11] Veena P.H., Pravin V.K. and Nagabhushan Joshi K. (2010): MHD visco-elastic flow and heat transfer with temperature gradient dependent heat skin in a porous medium past a stretching sheet. - IJMA, vol.3, No.1, pp.65-81.

[12] Gupta P.S. and Gupta A.S. (1977): Heat and mass transfer on a stretching sheet with suction or blowing. - Can. J. Chem. Engg., vol.55, pp.744-746.

[13] Kumaran V. and Ramanaiah G. (1996): A note on the flow over a stretching sheet. - Acta. Mech., vol.116, pp.229-233.

[14] McCormack D.D. and Crane L.J. (1973): Physical of Fluid Dynamics. - New York: Academic Press.

[15] Rajagopal K.R., Na T.Y. and Gupta A.S. (1984): Flow of a visco-elastic fluid over a stretching sheet. - Rheol. Acta., vol.23, pp.213-221.

[16] Pavlov K.B. (1974) : Magnetohydrodynamic flow of an incompressible viscous fluid caused by deformation of a plane surface. - Magnitanaya Gidrodinamika, vol.4, pp.146-147.

[17] Sarpakaya T. (1961): Flow of non-Newtonian fluids in a magnetic field. - AICHE, vol.7, pp.324-328.

[18] Dandapat B.S. and Gupta A.S. (1980): Flow and heat transfer in a visco-elastic fluid over a stretching sheet. - Int. J. Nonlinear Mech., vol.24, pp.215-219.

[19] Abel Subhash and Veena P.H. (1998): Visco-elastic fluid flow and heat transfer in a porous medium over a stretching sheet. - Int. J. Nonlinear Mechanics, vol.33, No.3, pp.531-543.

[20] Veena P.H., Pravin V.K., Shahjahan S.M. and Hippargi V.B. (2007): Non-similar solutions for heat and mass transfer flow in an electrically conducting visco-elastic fluid over a stretching sheet embedded in a porous medium. - IJMM, vol.2, No.1, pp.9-26.

[21] Veena P.H., Pravin V.K. and Padashetty S.C. (2007): Non-similar solutions in visco-elastic MHD flow and heat transfer over a stretching sheet with internal heat generation and stress work. - IJMM, vol.2, No.2, pp.267-281.

[22] Khan S.K. and Sanjayanand E. (2004): Visco-elastic boundary layer flow and heat transfer over an exponential stretching sheet. - Int. J. Heat Mass Transfer, vol.48, pp.1534-1542.

[23] Sajid M., Hayat T. and Asgar S. (2007): Non-similar analytical solutions for MHD flow and heat transfer a third order fluid over a stretching sheet. - Int. J. Heat Mass Transfer, vol.50, pp.1723-1736.

[24] Abel M.S., Sanjayanand E. and Nandeppanvar M. (2008): Visco-elastic MHD flow and heat transfer over a stretching sheet with viscous and ohmic dissipations. - Comm. In nonlinear Sci. and Numerical Simulation, vol.13, pp.1808-1821. 
[25] Panto Kratoras A. (2008): Study of MHD boundary layer flow over a heated stretching sheet with variable viscosity. - Int. J. Heat Mass Trans., vol.51, pp.104-110.

[26] Coleman B.D. and Noll W. (1960): An approximation theorem for functionals with applications in continuum mechanics. - Arch. Rat. Mech. Anal., vol.6, pp.355-370.

[27] Beard D.W. and Walters K. (1964): Proceedings Comb. Phil. Society, vol.60, p.667.

[28] Tak S.S. and Lodha A. (2005): Flow and heat transfer due to a stretching porous surface in presence of transverse magnetic field. - Acta. Chemica India, XXXIM3, pp.657-663.

[29] Rao B.N. (1996): Technical Note: Flow of a fluid of second grade over a stretching sheet. - Int. J. Non-Linear Mechanics, vol.31, No.4, pp.547-550.

Received: December 26, 2015

Revised: April 7, 2017 